\title{
Lense-Thirring QPO model testing by QPO phenomena in GX339-4 2010 outburst
}

\author{
H. Q. Gao, J. L. Qu, Z. Zhang and J. N. Zhou \\ Institute of High Energy Physics, CAS, Beijing 100049, P.R. China \\ email: gaohq@ihep.ac.cn
}

\begin{abstract}
Lense-Thirring QPO model is a promising model to explain QPO phenomena (Ingram et al. (2009)). In this model the QPO results from Lense-Thirring precession of a optical translucent inner hot flow in a truncated disc geometry. Now we check this model with different types QPO (see (Belloni et al. (2011)) for a recent review) of black hole transient (BHT) GX 339-4 2010 outburst and suggest type C QPOs are mainly coincident with this model prediction while type B QPOs are not.
\end{abstract}

Keywords. Black hole transient, Lense-Thirring precession, Quasi-periodic oscillations (QPO)

\section{Observations and data analysis}

We used only Proportional Counter Unit 2 from the RXTE PCA data since this unit was on during all the observations. The background was also extracted from Standard 2 data to correct PCU2 rates. In hardness analysis, we chose absolute channel bands $\mathrm{A}=$ 0-57 (2.06 - 24.00 keV), B = 0-13 (2.06 - 5.71 keV) and $\mathrm{C}=14-57(6.12-24.00 \mathrm{keV})$. A and $\mathrm{H}=(\mathrm{C} / \mathrm{B})$ were the total count rate and hardness respectively.

We used EVEnT mode data for timing analysis. Power density spectra (PDS) was produced for each observation using POWSPEC. The PDS was normalized though Miyamoto method (Miyamoto et al. (1991)) and the Poissonian noise was subtracted. For all the QPOs PDS, we used uniform model (multi lorentzian component for type C QPOs PDS; powerlaw component for band-limited noise and lorentzian component for type B QPOs PDS) to get the best fit.

\section{Result}

As the source flux increases, the truncation radius moves inwards and the hardness decreases. Lense-Thirring QPO model suggests a global precession of the entire hot flow which is a continuous progress. It takes place in the triangle points region where the type C QPO presents continuously shown in Fig. 1 the left panel. However the type B QPO locates at the peak of the flux and presents discontinuously make it can hardly be explain in this scenario. The Lense-Thirring model predicted the precession frequency to be dependent on the outer radius of the flow (see Ingram et al. (2009) Fig. 5). As the outer radius of the hot inner flow decreases from $50 R_{g}$ to several $R_{g}$, the QPO frequency moves from $0.1-10 \mathrm{~Hz}$ monotonously. Since it is difficult to define the outer radius based on RXTE data precisely, we use hardness change to represent the inward moving of the truncated disc radius during the source states transition. Comparing Fig. 1 right panel and the model prediction, we find the type $\mathrm{C}$ QPO frequency evolution are coincident with Lense-Thirring frequency change. But when the type B QPO appears, the frequency reaches a turning point and goes down as the hardness increases. All the type B QPOs locate in a small range of hardness indicates that the truncated disc radius 

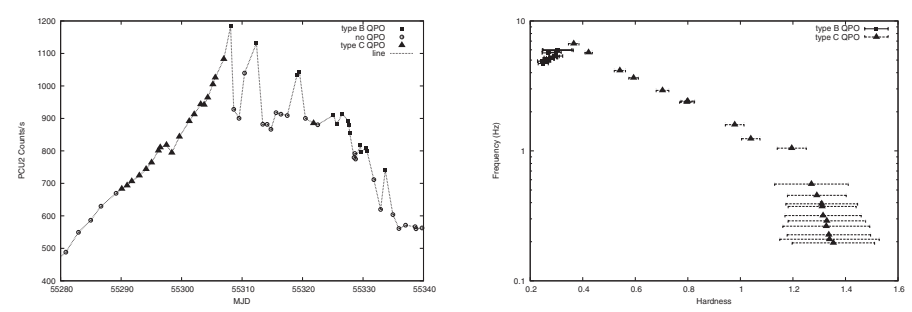

Figure 1. The left panel shows the lightcurve of GX339-4 2010 outburst rising phase during MJD 55280 to 55340. Each point stand for a PCA observation. The triangle one is the observation containing type $\mathrm{C}$ QPO, the square one is for type $\mathrm{B}$ and the hollow circular one has no QPO phenomena. the right panel shows QPO frequency versus Hardness. The triangle point is type $\mathrm{C} \mathrm{QPO}$ and the square one stands for type B QPO.
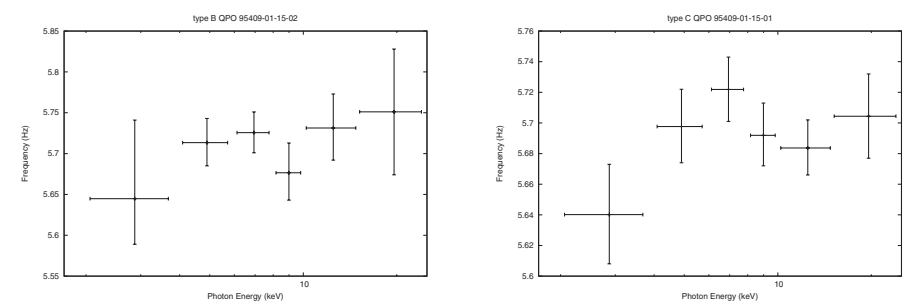

Figure 2. The QPO frequency versus photon energy for a type B QPO (left) and a type C QPO (right).

has no significant change. Combining the discontinuously appearance of type B QPO, it indicates some other mechanism begin to dominate the type B QPO phenomena. Fig. 2 left panel shows, in the different energy band photons, the type B QPO can keep in a uniform frequency range with the fitting uncertainties suggests the oscillation of type B $\mathrm{QPO}$ is from a quite compact region.

Since Lense-Thirring frequency is a global frequency of the hot inner flow treated as a rigid body in the model, it will produce a uniform frequency from the whole configuration. Fig. 2 right panel shows the type C QPO has significant frequency change below $7 \mathrm{keV}$ and almost remains stable above $7 \mathrm{kev}$. It indicates the Lense-Thirring precession of the hot inner flow may have more detail structure to produce the type C QPO.

\section{Conclusion}

We find that Lense-Thirring QPO model can mainly match the type C QPO in GX 339-4 2010 outburst but fail in type B QPOs because discontinuous appearance and frequency evolution. The hot inner flow may not keep absolutely rigid during precession to generate the type $\mathrm{C}$ QPO.

This work is supported in part by the National Natural Science Foundation of China, 973 program 2009CB824800 and NSFC-11173024.

\section{References}

Belloni, T., Motta, S., \& Munoz-Darias, T. 2011, BASI, 39, 409

Ingram, A., Chris, D., \& Chris, Fragile P. 2009, MNRAS, 397, L101

Miyamoto, S., Kimura, K., \& Kitamoto, S. 1991, ApJ, 383, 784

Motta, S., Munoz-Darias, T., Casella, P., Belloni, T., \& Homan, J. 2011, MNRAS, 418, 2292 